\title{
Collective Spontaneous Emission from a System of Quantum Dots
}

\author{
W. Abdussalam and P. MachnikOWSKi* \\ Institute of Physics, Wrocław University of Technology, Wybrzeże Wyspiańskiego 27, 50-370 Wrocław, Poland \\ We study the spontaneous emission from a regular lateral array or a randomly distributed ensemble of quan- \\ tum dots under strong excitation (full inversion) conditions. We focus on the similarities and differences between \\ the cases of random and regular arrangement of the dots and show that there is very little difference between \\ the evolution of luminescence in these two cases, both for identical dots and for a realistically inhomogeneously \\ broadened ensemble. This means that the enhanced emission or superradiance effect is not due to accidental \\ clustering of pairs of dots. Moreover, we point out that observation of an enhanced emission under weak excitation \\ does not prove that true superradiance will develop in a fully inverted system.
}

PACS: 78.67.Hc, 42.50.Ct, 03.65.Yz

\section{Introduction}

The phenomenon of collectively enhanced emission, described by Dicke for ensembles of atoms [1], can also occur in quantum dots (QDs) [2]. The cooperative radiation effect is due to the collective interaction of the two emitters with quantum radiation field. In the case of non-identical dots and in the absence of interactions, the collective emission appears if the interband transition energies in various QDs differ by no more than the emission line width [3], which requires the dots to be nearly identical, beyond the recent technological possibilities. However, coupling between the dots restores the collective nature of the emission and leads to accelerated or slowed down emission even for dots with different transitions energies [4].

Two typical couplings that may appear in a system of QDs are long-range Coulomb interactions and short-range couplings that may result from a combination of carrier tunneling and the Coulomb correlation effects. As we have shown, these two couplings show no qualitative difference in either accelerating or slowing down the emission in spite of their essentially different physical origin and properties [5]. The simulations of spontaneous emission from ensembles of coupled QDs show that the emission rate in such a system can indeed be increased due to collective coupling of the emitters to the electromagnetic field [6]. As the positions of the dots are random within the sample plane, it might happen that this enhanced emission effect results from clustering of dots in a self-assembled sample, as a result of which strongly coupled pairs of dots are formed and contribute to the emission. Therefore, in order to fully understand the role of the inter-dot coupling it is interesting to study the role of the planar arrangement of the dots on the observed collective emission. Moreover, so far the collective emission from QD ensembles was discussed only under weak excitation conditions (one excitation delocalized over the ensemble) [6], where it is manifested only in the form

* corresponding author; e-mail: Pawel.Machnikowski@pwr.wroc.pl of an enhanced emission rate. In order to observe actual superradiance, that is, a non-monotonic evolution of the emission with a delayed outburst of radiation [7], one has to study the photon emission under strong excitation conditions.

In this paper, we study the collective spontaneous emission from small ensembles of QDs under strong excitation (full inversion) conditions for two kinds of spatial arrangements of the QDs in the sample plane: regular arrays and randomly scattered dots. We show that the dynamics of emission very weakly depends on the QD arrangement.

The paper is organized as follows. In Sect. 2, we describe the model of a small ensemble of QDs in either random or regular planar arrangement. Next, in Sect. 3, we present and discuss the simulation results for the photon emission in these two cases. Finally, Sect. 4 concludes the paper.

\section{Model}

We consider an ensemble of a few (up to six) QDs placed in the $x y$ plane at positions $\boldsymbol{r}_{\alpha}$, where $\alpha$ numbers the dots. Each QD is modeled as a two-level system (empty dot and one exciton) with the fundamental transition energy $E_{\alpha}=\bar{E}+\epsilon_{\alpha}$, where $\bar{E}$ is the average transition energy in the ensemble. We consider two different arrangements of the QDs in the sample plane: regular arrays of QDs (double dots, triangular, rectangular aligned structure of the dots, penta- and hexagonal structures, see Fig. 1a) and ensembles of randomly distributed QDs, as schematically depicted in Fig. 1b. In the latter case, the ensemble is randomly distributed with a fixed surface density over square mesas with the restriction that the inter-dot distance cannot be smaller than $10 \mathrm{~nm}$. The spectral properties of the dots are modeled by a Gaussian distribution of their transition energies $E_{\alpha}$ with the standard deviation $\sigma$ around the mean $\bar{E}$. The dots are coupled by an interaction $V_{\alpha \beta}$ which can be either of dipole-dipole character (long-range dispersion force) or short-range (exponentially decaying with the distance). 


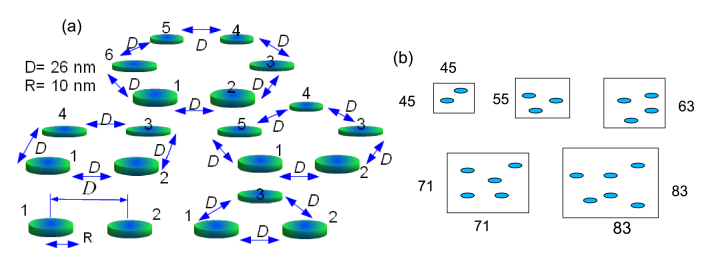

Fig. 1. The planar arrangement of the QDs: (a) regular array, (b) random ensemble (sample dimensions in nanometers).

We introduce the transition operators for the dots: the "exciton annihilation" operators $\sigma_{\alpha}$ which annihilate an exciton in the $\operatorname{dot} \alpha$, and the "exciton creation" operators $\sigma_{\alpha}^{\dagger}$ which create an exciton in the $\operatorname{dot} \alpha$. The exciton number operator for the $\operatorname{dot} \alpha$ is then $\hat{n}_{\alpha}=\sigma_{\alpha}^{\dagger} \sigma_{\alpha}$. Using these operators, the Hamiltonian of the many dots system is written in the frame rotating with the frequency $\bar{E} / \hbar$ in the form

$$
H=\sum_{\alpha=1}^{N} \epsilon_{\alpha} \sigma_{\alpha}^{\dagger} \sigma_{\alpha}+\sum_{\alpha \neq \beta=1}^{N} V_{\alpha \beta} \sigma_{\alpha}^{\dagger} \sigma_{\beta} .
$$

The long-range dipole coupling is described by

$$
V_{\alpha \beta}=V_{\alpha \beta}^{(\operatorname{lr})}=-\hbar \Gamma_{0} G\left(k_{0} r_{\alpha \beta}\right),
$$

where $\boldsymbol{r}_{\alpha \beta}=\boldsymbol{r}_{\alpha}-\boldsymbol{r}_{\beta}$,

$$
\Gamma_{0}=\frac{\left|d_{0}\right|^{2} k_{0}^{3}}{3 \pi \varepsilon_{0} \varepsilon_{\mathrm{r}}}
$$

is the spontaneous emission (radiative recombination) rate for a single dot, $\varepsilon_{0}$ is the vacuum permittivity, $\varepsilon_{\mathrm{r}}$ is the relative dielectric constant of the semiconductor, $k_{0}=n \bar{E} /(\hbar c)$, where $c$ is the speed of light and $n=\sqrt{\varepsilon_{\mathrm{r}}}$ is the refractive index of the semiconductor, and

$$
\begin{aligned}
& G(x)=\frac{3}{4}\left[-\left(1-\left|\hat{\boldsymbol{d}} \cdot \hat{\boldsymbol{r}}_{\alpha \beta}\right|^{2}\right) \frac{\cos x}{x}\right. \\
& \left.+\left(1-3\left|\hat{\boldsymbol{d}} \cdot \hat{\boldsymbol{r}}_{\alpha \beta}\right|^{2}\right)\left(\frac{\sin x}{x^{2}}+\frac{\cos x}{x^{3}}\right)\right],
\end{aligned}
$$

where $\hat{\boldsymbol{r}}_{\alpha \beta}=\boldsymbol{r}_{\alpha \beta} / r_{\alpha \beta}$ and $\hat{\boldsymbol{d}}=\boldsymbol{d} / d$, where $\boldsymbol{d}$ is the interband matrix element of the dipole moment operator which is assumed identical for both dots. For a heavy hole exciton, $\boldsymbol{d}=\left(d_{0} / \sqrt{2}\right)[1, \pm \mathrm{i}, 0]^{\mathrm{T}}$, so that for a vector $\boldsymbol{r}_{\alpha \beta}$ in the $x y$ plane one has $\left|\hat{\boldsymbol{d}} \cdot \hat{\boldsymbol{r}}_{\alpha \beta}\right|^{2}=1 / 2$. The short-range coupling is described by

$$
V_{\alpha \beta}=V_{\alpha \beta}^{(\mathrm{sr})}=V_{0} \mathrm{e}^{-r_{\alpha \beta} / r_{0}} \text {. }
$$

The effect of the coupling to the radiation field is accounted for by including the dissipative term in the evolution equations, which describes radiative recombination of excitons. The equation of evolution of the density matrix is then given by [8]:

$$
\dot{\rho}=-\frac{\mathrm{i}}{\hbar}\left[H_{0}, \rho\right]+\sum_{\alpha, \beta=1}^{N} \Gamma_{\alpha \beta}\left[\sigma_{\alpha} \rho \sigma_{\beta}^{\dagger}-\frac{1}{2}\left\{\sigma_{\alpha}^{\dagger} \sigma_{\beta}, \rho\right\}_{+}\right]
$$

where $\Gamma_{\alpha \alpha}=\Gamma_{\beta \beta}=\Gamma_{0}, \Gamma_{\alpha \beta}=\Gamma_{\beta \alpha}=\Gamma_{0} F\left(k_{0} r_{\alpha \beta}\right)$, with

$$
\begin{aligned}
& F(x)=\frac{3}{2}\left[\left(1-\left|\hat{\boldsymbol{d}} \cdot \hat{\boldsymbol{r}}_{\alpha \beta}\right|^{2}\right) \frac{\sin x}{x}\right. \\
& \left.+\left(1-3\left|\hat{\boldsymbol{d}} \cdot \hat{\boldsymbol{r}}_{\alpha \beta}\right|^{2}\right)\left(\frac{\cos x}{x^{2}}-\frac{\sin x}{x^{3}}\right)\right],
\end{aligned}
$$

and $\{\ldots, \ldots\}_{+}$denotes the anticommutator. The diagonal decay rates $\Gamma_{\alpha \alpha}$ describe the emission properties from a single dot, while the off-diagonal terms $\Gamma_{\alpha \beta}, \alpha \neq \beta$, account for the interference of emission amplitudes resulting from the interaction with a common reservoir and are responsible for the collective effects in the emission. The values of the two couplings as well as the interference term of the decay rate $\Gamma_{\alpha \beta}$ are plotted as a function of the distance between the dots in Fig. 2 .
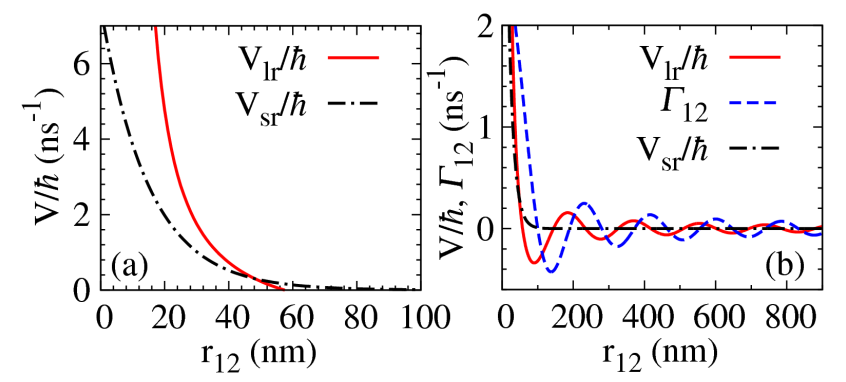

Fig. 2. The interference term of the decay rate $\Gamma_{12}$ and the short- and long-range coupling amplitudes $V_{\mathrm{lr}}, V_{\mathrm{sr}}$ as a function of the inter-dot distance. In (a), the small distance section is shown, while in (b) the oscillating tail at larger distances is visible.

In our simulations, we use the parameters for a typical CdSe/ZnSe QD system: $\Gamma_{0}=2.56 \mathrm{~ns}^{-1}, n=2.6$, the average transition energy of the QD ensemble $\bar{E}=2.59 \mathrm{eV}$ and the QD surface density $\nu=10^{11} \mathrm{QDs} / \mathrm{cm}^{2}$. For the tunnel coupling we choose the amplitude $V_{0}=5 \mathrm{meV}$ and the range $r_{0}=15 \mathrm{~nm}$.

\section{Results}

In Fig. 3 we show the results of numerical simulation based on Eq. (1) for regular QD arrays. At the initial time, the system is strongly excited to the fully inverted state $|\psi(0)\rangle=|1 \ldots 1 \ldots 1\rangle$ and we subsequently determine the photon emission rate as a function of time. On each plot, the photon emission in the system is shown as a function of time for identical dots $(\sigma=0)$ and for non-identical dots with the realistic value of the fundamental transition energy standard deviation $\sigma=18.4 \mathrm{meV}$.

As can be seen in Figs. 3a and b, the spontaneous emission from a regular array of identical QDs develops a maximum, which is a few-emitter counterpart of the delayed outburst of photon emission observed in macroscopic samples [7]. This effect appears already for three QDs and becomes more and more pronounced as the number of dots increases. It implies that the true superradiance effect appears in the regularly arranged small ensemble of identical QDs with either long- or short-range couplings. However, if the array is formed by 


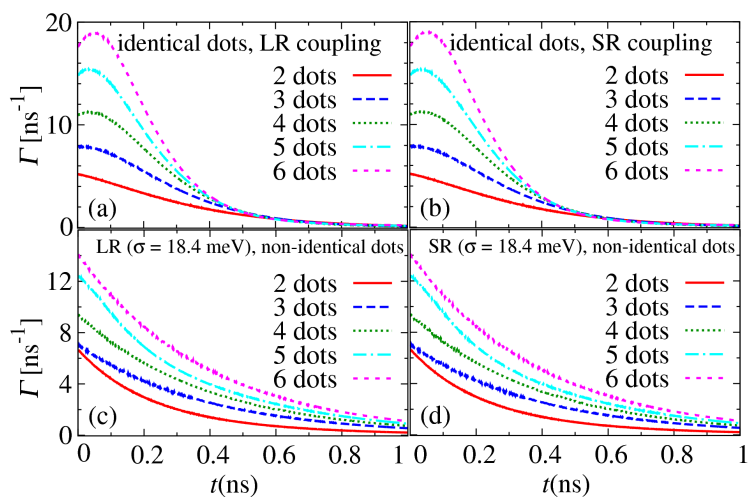

Fig. 3. The evolution of photon emission for the fully inverted initial state in regular arrays of a few QDs.

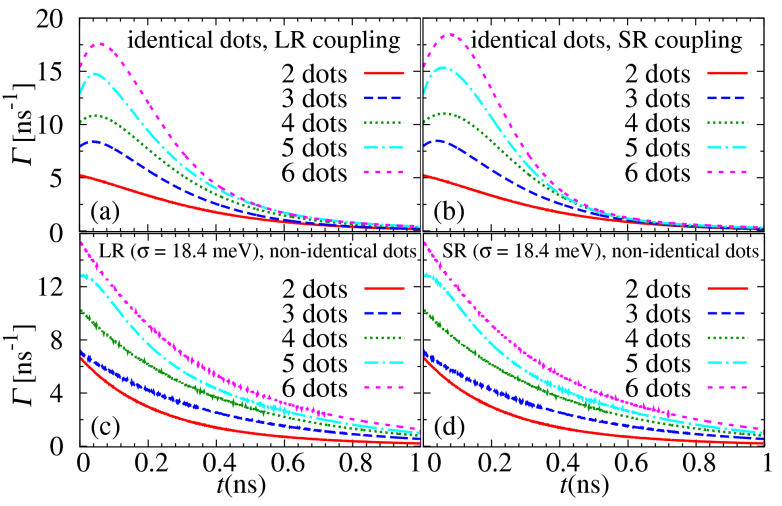

Fig. 4. The evolution of photon emission for the fully inverted initial state in an ensemble of a few QDs with a random spatial distribution.

non-identical dots with a realistic inhomogeneity of the fundamental transition energies (Fig. 3c and d), when the value of $\sigma$ is greater than both the coupling strengths and the inverse exciton life time (the emission line width), then the maximum is completely washed out. Thus, under conditions that led to enhanced emission in the weak excitation regime [6], full superradiance effects under strong excitation are not observed.

In order to find out whether a randomly distributed ensemble of QDs behaves similar to a regular array of QDs we now study the photon emission from a random QD ensemble in the same strong excitation regime. In each case, we calculate an average of 100 realizations of the evolution in systems with a given number of dots but differing in their positions within the sample plane. Figure $4 \mathrm{a}$ and $\mathrm{b}$ shows the simulated photon emission for an ensemble of identical dots. Again, a superradiant maximum builds up as the number of QDs grows. Similarly to the case of regular QD arrays, the maximum disappears and the decay of luminescence becomes monotonic when the realistic distribution of fundamental transition energies is taken into account (Fig. 4c and d).

By comparing Fig. 3 with Fig. 4 one can see that there is very little difference in arranging the dots either randomly or regularly under strong excitation conditions. Regular ordering of QDs only slightly enhances the peak emission in the hypothetical case of identical dots and slightly suppresses the emission in the realistic case of an inhomogeneously broadened ensemble.

\section{Conclusions}

We have shown that the delayed outburst of radiation, typical for the superradiant emission develops in the luminescence from regular arrays or randomly distributed ensembles of quantum dots in the strong excitation regime. The way the dots are distributed in the sample plane (regular vs. random) makes very little difference on the photon emission. This means that the system response is not dominated by accidental clustering that might appear in the random distribution case and lead to strongly enhanced contribution to the overall emission from pairs of accidentally very closely spaced dots. Moreover, in both cases, the superradiant maximum is completely washed out if the realistic degree of inhomogeneity of the fundamental transition energies is taken into account. Thus, enhanced emission observed experimentally under weak excitation does not imply that true superradiance will be manifested for a fully inverted system.

\section{Acknowledgments}

This work was supported by the Foundation for Polish Science under the TEAM programme, co-financed by the European Regional Development Fund.

\section{References}

[1] R.H. Dicke, Phys. Rev. 93, 99 (1954).

[2] M. Scheibner, T. Schmidt, L. Worschech, A. Forchel, G. Bacher, T. Passow, D. Hommel, Nat. Phys. 3, 106 (2007).

[3] A. Sitek, P. Machnikowski, Phys. Rev. B 75, 035328 (2007).

[4] A. Sitek, P. Machnikowski, Phys. Rev. B 80, 115301 (2009); 80, 115319 (2009).

[5] W. Abdussalam, P. Machnikowski, Acta Phys. Pol. A 120, 865 (2011).

[6] M. Kozub, Ł. Pawicki, P. Machnikowski, Phys. Rev. $B$ 86, 121305R (2012).

[7] N. Skribanowitz, I.P. Herman, J.C. MacGilvray, M.S. Feld, Phys. Rev. Lett. 30, 309 (1973).

[8] R.H. Lehmberg, Phys. Rev. A 2, 883 (1970); R.H. Lehmberg, Phys. Rev. A 2, 889 (1970). 